\title{
A Holey Fiber-Based Nonlinear Thresholding Device for Optical CDMA Receiver Performance Enhancement
}

\author{
J. H. Lee, Student Member, IEEE, P. C. Teh, Student Member, IEEE, Z. Yusoff, Student Member, IEEE, M. Ibsen, \\ W. Belardi, T. M. Monro, and D. J. Richardson
}

\begin{abstract}
In this letter, we demonstrate the use of an optical thresholder-based on a short length of holey fiber to achieve enhanced code recognition quality in a 255-chip 320-Gchip/s superstructured fiber Bragg grating-based optical code-division multiple access code : decode system. The nonlinear thresholder is based on bandpass filtering of spectrally broadened components generated by self-phase modulation and assisted by the Raman effect in an $8.7 \mathrm{~m}$ length of highly nonlinear holey fiber. Error free penalty free system performance is obtained with complete recovery of the original input pulse shape.
\end{abstract}

Index Terms-Gratings, optical code-division multiaccess, optical fiber communication, optical fiber devices, optical signal processing, optical switching.

\section{INTRODUCTION}

$\mathbf{T}$ HERE IS A GROWING interest in the development of all-optical code generation and recognition techniques for optical code-division multiple access (OCDMA) applications. Optical matched filtering using pairs of optical filters with conjugate impulse response functions provides perhaps the most convenient means to generate/recognize optical code sequences. Recently, superstructured fiber Bragg grating (SSFBG) technology has emerged as a means of fabricating filters with suitably precise and complex impulse response functions for generating/recognizing both amplitude and phase encoded sequences [1]. In SSFBG-based OCDMA schemes, the reflection of a short pulse from one SSFBG (the encoder) results in the generation of a code sequence. This code can then be recognized (at a particular receiver) by subsequent reflection from a second SSFBG (the decoder) that has a spatially reversed refractive index profile relative to that of the encoder. This process results in the generation of a pulse form in the time domain with a temporal structure corresponding to the autocorrelation function of the code. Simple intensity discrimination techniques can then be used to detect code recognition provided that only code sequences are used within the system that have: a) a distinctive autocorrelation characteristic comprising a short, relatively intense pulsed feature, (which is in general unavoidably accompanied by an extended low level pedestal), and b) mutually low-cross correlation characteristics. Whilst good operation of such "linear"

\footnotetext{
Manuscript received October 24, 2001; revised December 21, 2001.

The authors are with the Optoelectronics Research Centre, University of Southampton, Highfield, SO17 1BJ Southampton, U.K. (e-mail: jhl@orc.soton.ac.uk).

Publisher Item Identifier S 1041-1135(02)04078-8.
}

optical systems can be achieved, nonlinear optical techniques that can eliminate the extended low-level pedestal associated with the matched filtering process are desirable, since removal of this pedestal enhances the contrast of the pattern recognition signature. This is particularly important within OCDMA systems since a particular coded signal generally needs to be recognized in the presence of other orthogonally coded signals, each of which gives also rise to low level pedestal signals when filtered, and which can interfere with the decoded signal.

The use of fast fiber-based nonlinear thresholding devices within OCDMA receivers has been previously demonstrated, and these devices were based on either the filtering of spectrally broadened signal components generated through Kerr nonlinearity [2], [3], or soliton effects in a nonlinear optical loop mirror [4]. Both of these devices employed conventional silica fibers and were impractical since the relatively low nonlinearity of these fiber types means that long fiber lengths (several kilometers) are required.

Holey fiber (HF) technology, however, now allows the fabrication of fibers with very tightly confined modes, and thus very high optical nonlinearities per unit length. Indeed, a silica holey fiber can have a nonlinearity 10 100 times that of a conventional silica fiber. Nonlinear devices based on HF can, thus in principle, be 10-100 times shorter than similar devices based on conventional fiber technology, offering a route to the development of truly practical, ultrafast fiber based nonlinear devices.

In this letter, we present for the first-time experimental results concerning the use of a HF nonlinear thresholding device within an OCDMA receiver. Using a short $(8.7 \mathrm{~m})$ length of HF, we achieve enhanced code recognition quality in a 255-chip 320-Gchip/s SSFBG based OCDMA code : decode system. Error free penalty free system performance is obtained and the $\sim 3-\mathrm{dB}$ power penalty observed for pure matched filtering alone is eliminated.

\section{EXPERIMENTAL SETUP AND RESULTS}

Our experimental setup is shown in Fig. 1(a). The 2.5-ps pulses at $10 \mathrm{GHz}$ are first generated using a regeneratively mode-locked erbium fiber ring laser (EFRL) operating at $1553 \mathrm{~nm}$. These are then gated down in frequency and then modulated to obtain a $1.25-\mathrm{Gb} / \mathrm{s}$ data stream. The $1.25-\mathrm{Gb} / \mathrm{s}$ short pulse data stream was then launched onto an SSFBG encoder (C1) containing 255-chip 320-Gchip/s quaternary phase code sequence coding information. The chip duration 


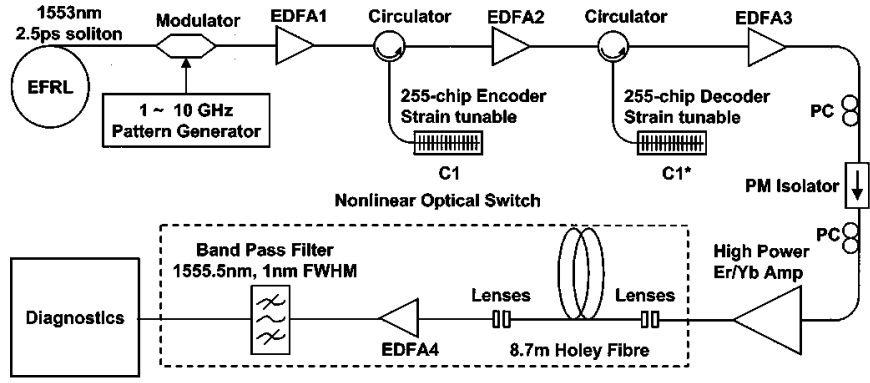

(a)
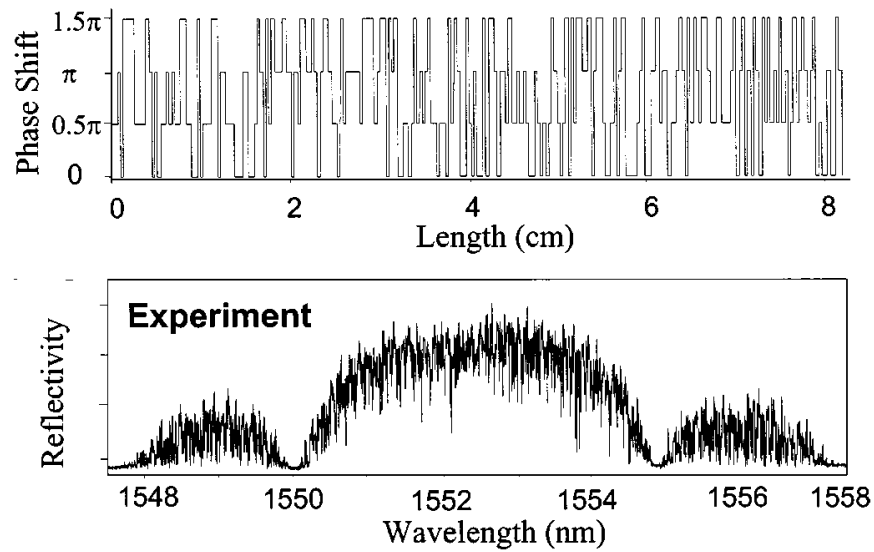

(b)

Fig. 1. (a) Experimental setup. (b) Four-level phase coding scheme and measured grating reflectivity profile.

was thus $3.2 \mathrm{ps}$ and the coded data pulses had a total duration of $\sim 800$ ps. The particular code as shown in Fig. 1(b) is a member of the Family $\mathcal{A}$ sequences that have been applied within wireless CDMA communications. The characteristics and properties of the gratings are more fully described in [1]. The coded signal was then fed directly to the associated matched decoder grating $\mathrm{C} 1 *$. Since the encoder and decoder gratings are matched then, as previously explained, the decoder output should exhibit a short, chip length long autocorrelation spike centered on a relatively broad, (1.6 ns) pedestal. The nonlinear HF-based optical thresholder was located after the decoder grating and was preceded by a fiber amplifier.

An SEM of the transverse profile of the highly nonlinear HF is shown inset in Fig. 2. The core diameter is $\sim 2.0 \mu \mathrm{m}$ and the outer diameter of this fiber is $125 \mu \mathrm{m}$. Due to the large air holes and small core size, the fiber is highly nonlinear and the asymmetric arrangement of holes around the core results in a very large linear birefringence. The measured beat length is $0.43 \mathrm{~mm}$, which is $\sim 10$ times shorter than that of commercially available PM fiber types. This means that fibers with extremely good polarization-maintaining characteristics can be fabricated using HF technology. From a measurement of the SPM-induced nonlinear phase shift versus launched optical power as shown in Fig. 2 [5], we obtained a value of $\gamma=31 W^{-1} \cdot \mathrm{km}^{-1}$, from which we derive an estimate of $A_{\text {eff }}=2.93( \pm 0.3) \mu \mathrm{m}^{2}$ for its effective area. This nonlinearity is $\sim 20$ times higher than that of a conventional dispersion shifted fiber.

Self-phase modulation (SPM) accompanied by Raman scattering in the highly nonlinear HF due to the intense autocorrelation spike results in (asymmetric) spectral broadening of

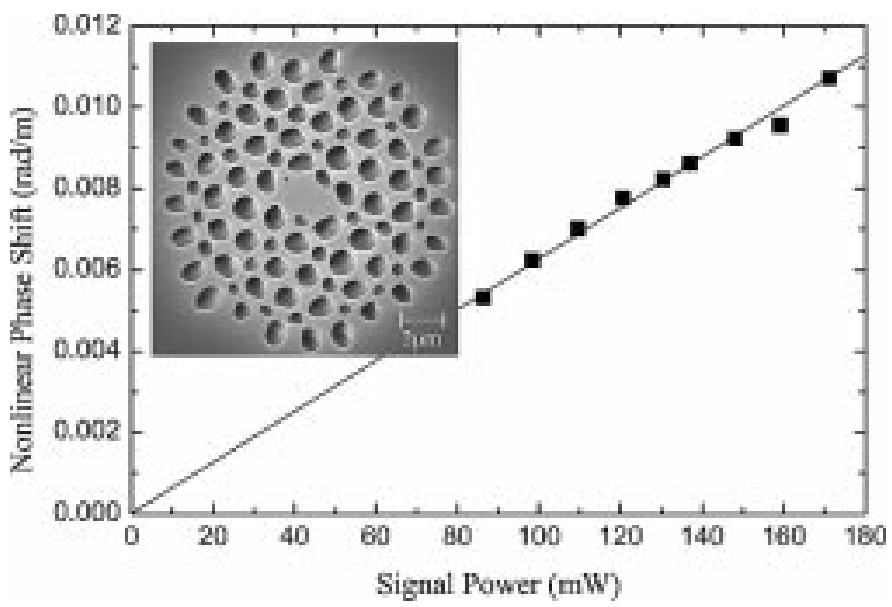

Fig. 2. Measured SPM-induced nonlinear phase shift versus launched optical power in the HF. Inset: Cross-sectional SEM image of the HF used.

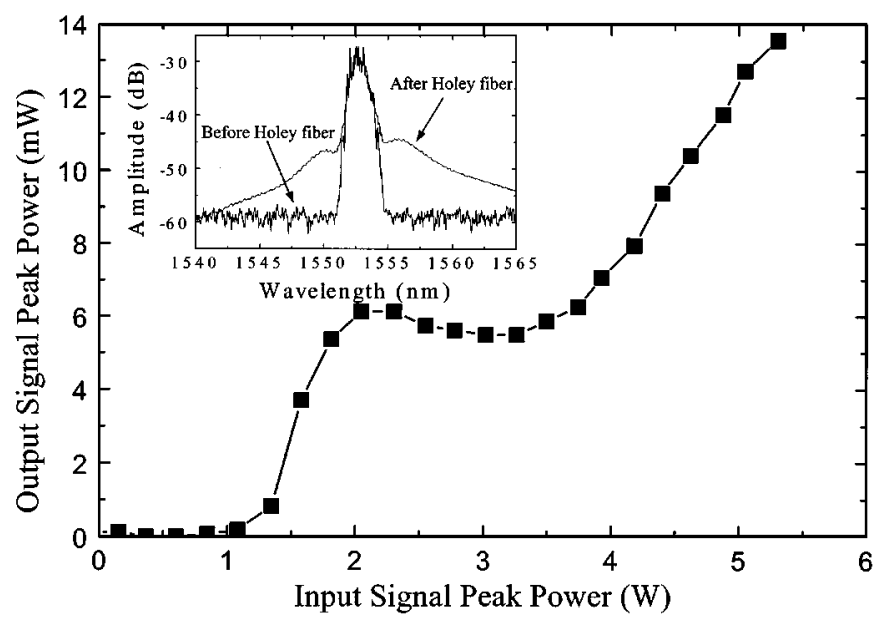

Fig. 3. Experimentally obtained power transmission characteristic of the HF switch as a function of the input peak power. Inset: Signal spectrum both before and after the $\mathrm{HF}$.

correctly decoded bits (see inset of Fig. 3). A self-switched signal with a transfer characteristic suitable for intensity thresholding as shown in Fig. 3 was obtained by filtering the resulting spectrally broadened signal with a narrow-band dielectric filter that had its center-wavelength offset by $(+2.5 \mathrm{~nm})$ relative to the peak wavelength of the incident signal. The power loss of $\sim 25 \mathrm{~dB}$ between input and output in the nonlinear switch comes from various origins including background HF loss $(\sim 0.5 \mathrm{~dB})$, input/output beam coupling (total $\sim 8-\mathrm{dB}$ loss), and of course the offset spectral filtering. The $3-\mathrm{dB}$ bandwidth of the filter was $1 \mathrm{~nm}$. This value was chosen to ensure that the switched output pulses had roughly the same temporal width as the 2.5-ps input pulses [see Fig. 4(a)].

In order to assess the impact of using the HF switch as an optical thresholder, we first measured the eye diagrams. The improved pattern recognition contrast is clearly evident on a 20-ps timescale by comparing the eyes and background noise levels in Fig. 4(b), and on a picosecond timescale in Fig. 4(a). The low-level pedestal obtained with simple matched filtering is almost completely eliminated. To quantify the benefits of the pedestal rejection from a system perspective bit error rate (BER) 


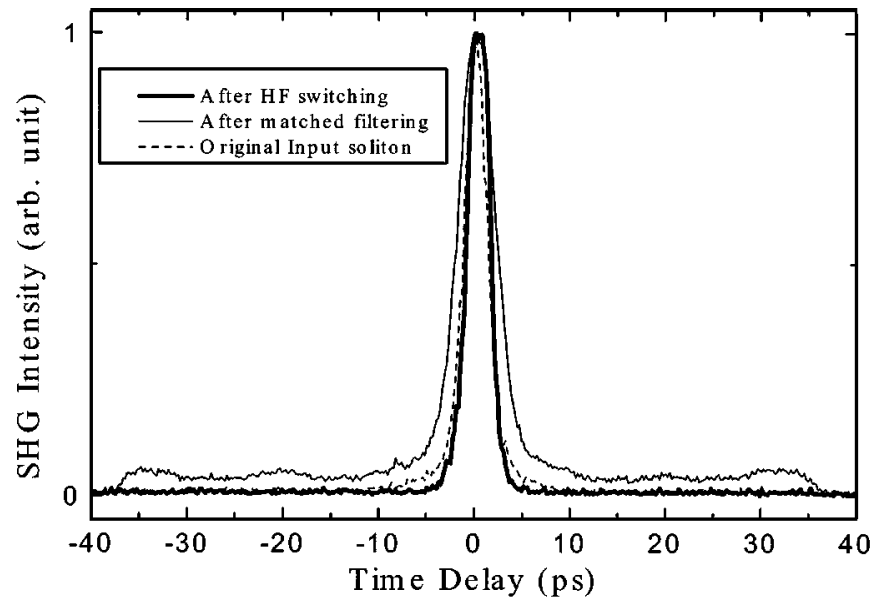

(a)
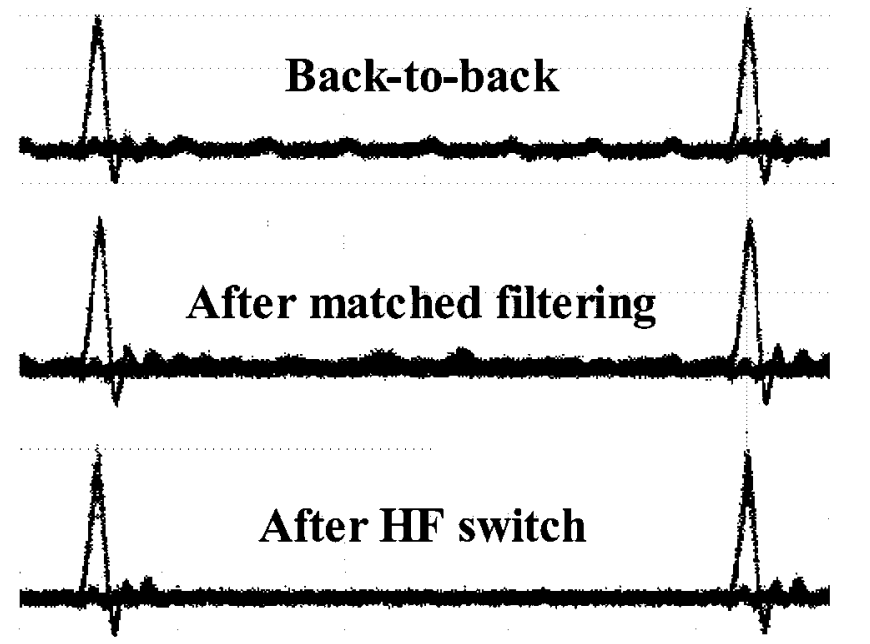

(b)

Fig. 4. (a) SHG autocorrelation traces. (b) Eye diagrams for the $1.25-\mathrm{Gb} / \mathrm{s}$ OCDMA system.

measurements were performed as shown in Fig. 5. The observed $\sim 3-\mathrm{dB}$ power penalty associated with matched filtering alone is totally eliminated. It should be mentioned that since the fiber has anomalous dispersion (D 100 ps/nm. $\mathrm{km}$ ) the filtering process results in additional noise on the "one" bits [see Fig. 4(b)] due to coherence degradation [6]. However, for this particular application this does not appear to lead to any penalty. Indeed, a $\sim 1$-dB penalty improvement relative to the laser back-to-back case was obtained due to clean up of residual radiation in the zero bit slots due to the poor $(\sim-14 \mathrm{~dB})$ extinction ratio of the external data modulator [see Fig. 4(b): back-to-back trace].

\section{CONCLUSION}

We have experimentally demonstrated that a nonlinear optical switch based on a short length of HF can be readily used to

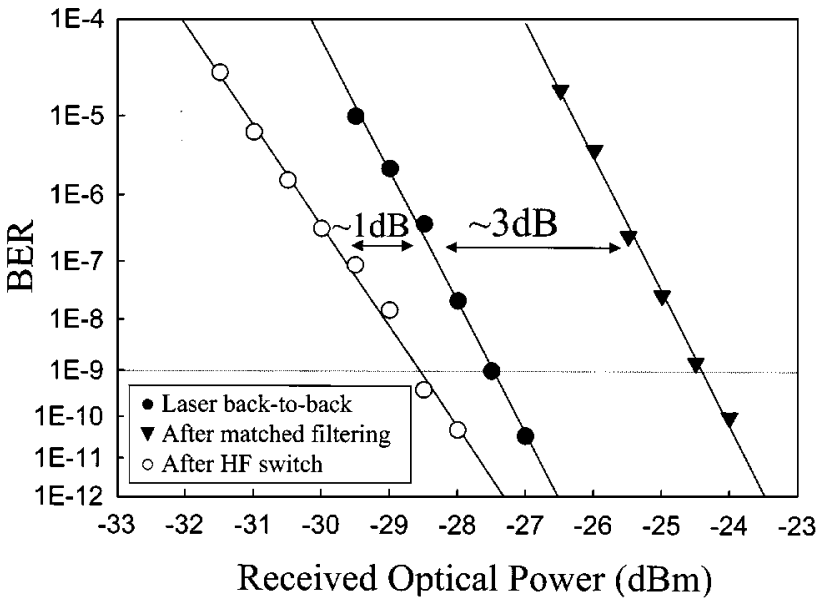

Fig. 5. Measured BER versus received optical power for the $1.25-\mathrm{Gb} / \mathrm{s}$ OCDMA system.

enhance code recognition contrast in a SSFBG-based OCDMA code : decode system. We believe that our HF-based nonlinear thresholding device can also be applied to improve the performance of multiuser OCDMA systems [4]. This experiment represents just one example of a practical application of a HF-based nonlinear device. We consider that this will be the first of many such demonstrations and that nonlinear HF devices will ultimately prove of great practical relevance for future communication systems.

\section{ACKNOWLEDGMENT}

The authors would like to thank Prof. A. Grudinin and Dr. S. Alam of Southampton Photonics Inc. for the loan of an $\mathrm{Er}-\mathrm{Yb}$ amplifier and associated help.

\section{REFERENCES}

[1] P. C. Teh, M. Ibsen, J. H. Lee, P. Petropoulos, and D. J. Richardson, "A 4-channel WDM/OCDMA system incorporating 255-chip, $320 \mathrm{Gchip} / \mathrm{s}$ quaternary phase coding and decoding gratings," in Proc. Optical Fiber Communications Conf. (OFC'2001), Anaheim, Mar. 2001, Postdeadline Paper PD37.

[2] P. V. Mamyshev, "All-optical data regeneration based on self-phase modulation effect," in Proc. ECOC'98, Madrid, Spain, Sept. 1998, pp. 475-476.

[3] H. P. Sardesai, C.-C. Chang, and A. M. Weiner, "A femtosecond codedivision multiple access communication system test bed," J. Lightwave Technol., vol. 16, pp. 1953-1964, Nov. 1998.

[4] J. H. Lee, P. C. Teh, P. Petropoulos, M. Ibsen, and D. J. Richardson, "Reduction of interchannel interference noise in a two-channel, grating based OCDMA system using a nonlinear optical loop mirror," IEEE Photon. Technol. Lett., vol. 13, pp. 529-531, May 2001.

[5] A. Boskovic, S. V. Chernikov, J. R. Taylor, L. Gruner-Nielsen, and O. A. Levring, "Direct continuous measurement of $\mathrm{n} 2$ in various types of telecommunication fiber at $1.55 \mu \mathrm{m}$," Opt. Lett., vol. 21, no. 24, pp. 1966-1968, 1996.

[6] N. Nakazawa, H. Kubota, and K. Tamura, "Random evolution and coherence degradation of a high-order optical soliton train in the presence of noise," Opt. Lett., vol. 24, no. 5, pp. 318-320, 1999. 